\title{
The development of visualization of transmedia storytelling projects with a tree structure
}

\author{
Shunsuke Aoki ${ }^{1}$, Takayuki Fujimoto ${ }^{2}$ \\ ${ }^{1}$ Graduate School of Information Sciences and Arts Toyo University, Tokyo, Japan (e-mail: \\ shunsuke.aoki.bw@gmail.com). \\ ${ }^{2}$ Graduate School of Information Sciences and Arts Toyo University, Tokyo, Japan (e-mail: fujimoto@toyo.jp)
}

Article History:Received:11 november 2020; Accepted: 27 December 2020; Published online: 05 April 2021

\begin{abstract}
In recent years, transmedia storytelling (TMS) technique is gatheringattention in Europe and America. Telling a story by using multiple kinds of media provides strong impression and immersivesensations with audiences. Therefore TMS technique has been incorporated in various fields.However, it is hard for creators to work on TMS project because of the complexity. The complexity is also an obstruction of popularization.In this study, by using the tree structure for TMS projects, we organize and visualize the complex content information of TMS. As a result, creators can work on a TMS project more efficiently. We analyze some successful TMS cases of different genres by applying them to a tree structure. Thereafter, we clarify what kind of effects creators can get by using a tree structure.
\end{abstract}

Keywords: Transmedia storytelling, transmedia, narratology, promotion

\section{BACKGROUND}

Various kinds of media platforms have been invented and developing along with rapid IT revolution.Today, media platforms are related to our life deeply and they are always inseparably close to us. In recent years, Transmedia storytelling (TMS), which is the approach technique to audience is gathering attention in Europe and America.TMS is the technique to deliver story experience to audiences by developing one story through some media platforms and expanding the story world.Nowadays, in a flood of diverse contents, the story that is conveyed through just in one kind of media platform is not as influential as the content that uses TMS technique. By utilizing some media platforms, audiences can approach to the story's various aspects with their different sensations. To succeed in a TMS project, creators need to think about the essentials factors for TMS project such as how to mutually combine distributed stories, which are on different media platforms, expecting audiences' response and how to control their actions.TMS project also, requires long-term based and detailed plan. It is not easy for creators who are not familiar with how TMS goes, to work on TMS project.TMS project works only when proper story is delivered to proper audiences with proper media platforms at proper timing

\section{Purpose}

TMS is a technique to approach to audiences by developing one story using some media platforms. The concept that the audience can enjoy story experiences is one of the TMS features.Audience can enjoy developed stories in each media with different sensations.On the other hand, because of TMS complexity, creators need detailed plan and enormous time to contrive the project.There is a tendency that TMS is easy to be confused with cross-media marketing and media-mix marketing. The effect of TMS technique has been verified and TMS is expected to develop further in the future. Taking them into consideration, it should be said that the TMS complexity is a barrier to the widespread use and correct understanding.In this study, by utilizing a tree structure for TMS project, we demonstrate that the image of the entire project can be visualized.Generally, a tree structure is used for the purpose of organizing information. The creators who work on TMS project can proceed with the project more efficiently by using a tree structure.We extract famous successfulcases from each of threekinds of main fields where TMS is used, and apply those cases to a tree structure.In this way, we reveal how the tree structure affecteach of the features of TMS projects. We also mention what kind of effects user can obtain from the tree structure as a result. III. WHAT IS TMS?

According to H. Jenkins, who is the leading expert of TMS, TMS is defined as "Transmedia storytelling (TMS) represents a process where integral elements of a fiction get dispersed systematically across multiple delivery channels for the purpose of creating a unified and coordinated entertainment experience. Ideally, each medium makes its own unique contribution to the unfolding of the story." (Jenkins 2007). Mainly, TMS tends to be used in the three kinds of industries.Firstly, one of them is 'advertising'.Famous companies such as Coca Cola and Heineken conducted the promotion campaigns using TMS technique. The advantageof TMS regardinga promotion campaign is ability of giving the strong impression of the product to the target audience. A campaign is not just developed in advertisement such as commercial films on TV and paper medium. The advertisers also use

*Corresponding author: 
Social Networking Service (SNS) applications and deliver story experiences to the targets. Secondly, another industry is 'Alternate Reality Game (ARG)'. The feature of ARG is that a player proceeds with a game while going back and forth between a real world and media. For example, one problem-solving game does not involve one medium as a video game, and the players can procced the story only when they can get a hint for the game on the dedicated website in the real world.By going back and forth between the 'real' and the media, the player gets the illusion that he or she is actually in the game.Lastly, the other industry is 'entertainment series'.Regarding entertainment series, one media content is set as the main story and the side story and the back story are developed in other media platforms.In the process of the development of the story, that new characters join the story and the story world expands. That is one of the reasons why the series can keep attracting the audience.

\section{WHAT IS TREE STRUCTURE?}

A tree structure is data structure that arranges and manages data by setting the hierarchy upon one data.It is called a tree structure because it has multiple children for one parent and it branches and spreads.Mainly, a tree structure is used to organize contents of data and websites systematically. As a general rule, once it branched, it is never combined with other branches like a real tree.There are various types of tree structures, and they are used differently according to the purposes.A "binary tree" is a tree whose number of children is limited to two, and a tree that can have three or more children is called a ' $\mathrm{m}$-ary tree'. A tree whose number of children is limited to $\mathrm{N}$ is generally called an ' $\mathrm{N}$-ary tree'.Moreover, there is also a 'balanced tree' constructed so that the height of the leaves is as equal as possible. Balance tree is often used for the purpose of shortening the average calculation time when performing processing such as search. "B tree" is a structure that is like m-ary tree and balanced tree.A tree structure is often used for file systems and databases.this structure is a quite effective to organize files. However, only with the tree structure, inconvenient problems happen to arrange files. To solve them, the systems such as 'alias, for Macintosh', 'shortcut, for Windows' and 'symbolic link, for UNIX' are utilized.In a tree structure, naturally there is only one way to reach one node. In the mechanism above, different names are given totwo nodes so that they can be reached from two ways (Shinjo, 2016).

Next, we mention about a university website as an example of a tree structure use.The parent of the tree structure is the top page of the website. 'Academics', 'About university' and 'International exchange'are in the top page and they are the children of the top page.Upon "Academics", there are "Faculties and Departments" and "Students support" which are detailed information, and they are the children of 'Academics' (Fig.1).In this way, by subdividing and organizing data from outline information to detailed information, users can easily find the information that they are looking for.

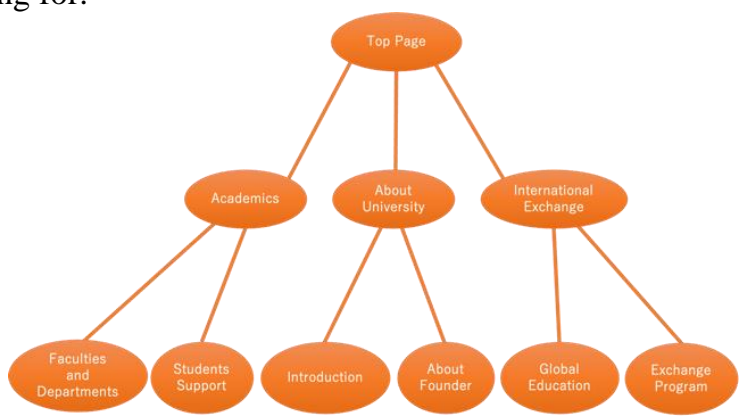

Fig. 1. Atree structure of a university website.

By applying this tree structure to TMS, it becomes easy for creators to catch the image of the whole project.In addition, a tree structure is also effective for the process to create and manage the project.All stories have 'a beginning'. The beginning of the story becomes the parent of the tree structure, and the story isbranched and developed through multiple media platforms, and then the story world expands. The creators can easily see how each content is related to each other and the whole balance.As mentioned previously, mainly, TMS technique is used for three kinds of fields (Entertainment series, ARG, and Advertising).

In the next chapter, we will apply the content of successful cases that represents each field to the tree structure, and explain how a tree structure is suitable for TMS projects.

\section{VISUALIZATION OF TMS PROJECT BY USING A TREE STRUCTURE}

\section{A. Entertainment series}

"Star wars" is one of the successful cases that used TMS technique as entertainment series. First film was released in 1977, after that the sequels were released one after another.

The impression of "Star wars" might be 'movie series' to the people who are not the fans.However, the story of "Star wars" has been developed in various media.The side stories have been developed and the story world is expanding with animation movies, TV animation series, comics and live-action drama series (Fig.2). 

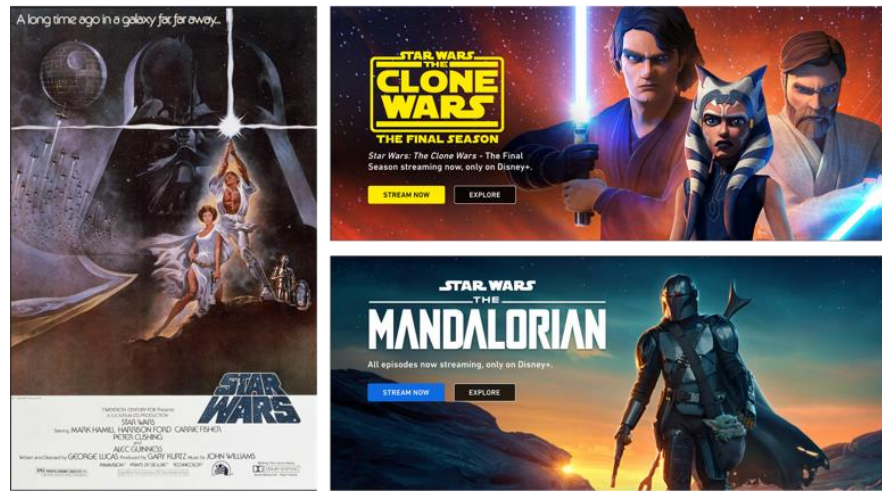

Fig.2.The various works of Star wars [5].

These multiple kinds of content attract people, and it can be said that this is one of the reasons to give the fans sense of immersion to the series. Creators create unique stories and story worlds to fascinate audiences. The remarkable feature of "Star wars" is complexity of timeline. The one firstly produced was Episode 4, and Episodes 1,2 , and 3 were released in the way to get back to the past.Side story was also developed in the animation series and the live-action drama series.According entertainment series, a tree structure can play a role as 'Time line' of a story (Fig.3).Furthermore, "Star wars" has a diversity regarding the themes of the story.It goes without saying that it involves action and suspense movie touch, and there are also various aspects such as a love story, social drama and family story. This is also one of the charms to make fans not to get bored. By using a tree structure, it is easy to figure out how various side stories are related to the main story.

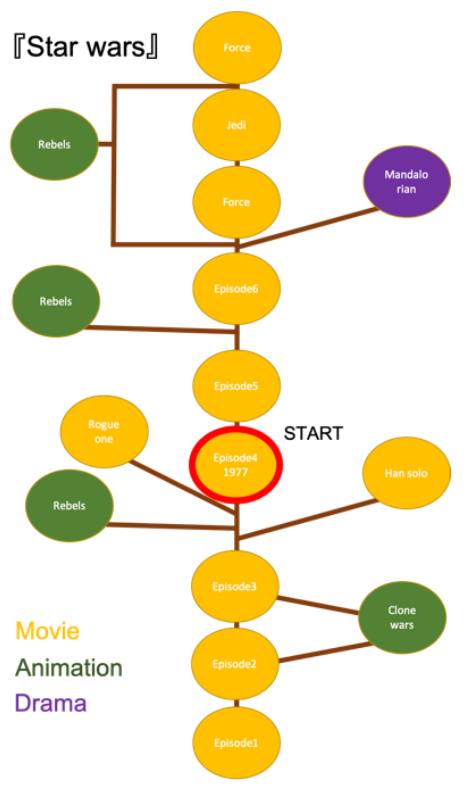

Fig.3.A tree structure of Star wars.

\section{B. Alternate Reality Game (ARG)}

The successful case that represents ARG is "Why so serious?", which are delivered as a promotion campaign of "The Dark Knight."As it literally suggests, ARG is a game, and the player involves with the story.For that reason, the creator needs to guide the players' actions properly and let the story goes as planned to make the players complete the story."Why so serous?" is the largest example in terms of both the variety of the gimmicks and the number of players.As for TMS project for ARG, by utilizing a tree structure, it becomes easy to figure out the relationship between 'Story' and 'Story experience'.In addition, by visualizing what kind of mechanism let the player advance to the next phase, it is possible for a creator to grasp the overall balance.In the case of "Why so serious?", 'Solving the mystery on the website' and 'Live event' are set up alternately (Fig.4). 

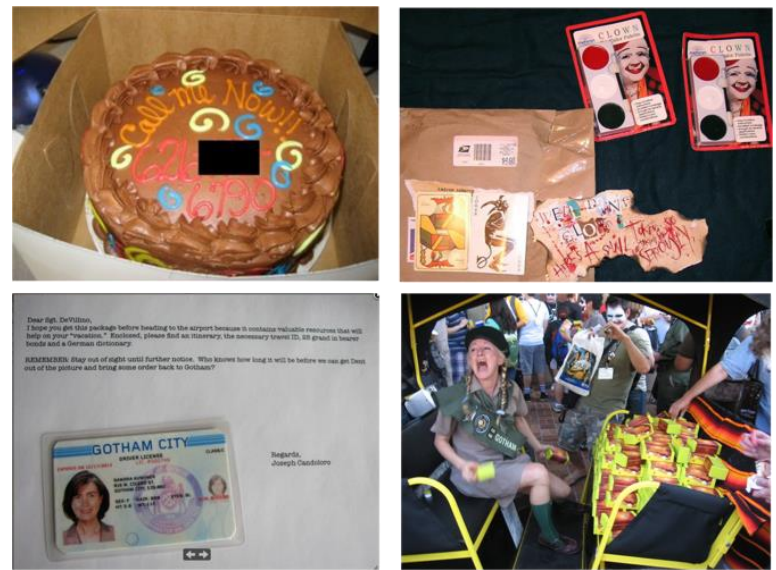

Fig.4.Dark Knight Campaign "Why so serious?” [6].

Live events can offerimmersive sensation and it is easy to come up as a topic of people's conversation, but the number of people who can participate in is limited.On the other hand, the problem-solving game of the website allows the players all over the world to play, but it cannot provide sense of immersion as much as that by the live event."Why so serious?" incorporated the good points of both live events and games on the website, and they controlled the player actions well without getting them bored (Fig.4). There is also a type of ARG upon which the development of the subsequent story changes depending on the player's choices in the game.For this type of ARG, by using a tree structure, the creators can figure out relationships between the player choices and the patterns of the story-ending easily.

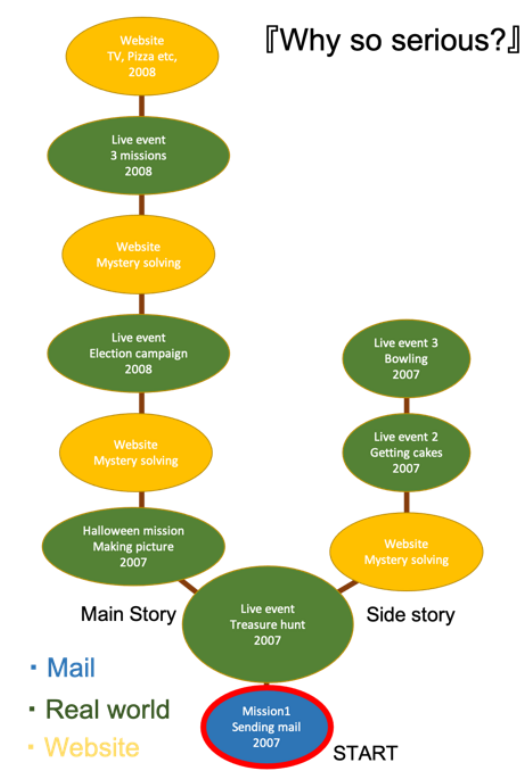

Fig.4.A tree structure of "Why so serious?".

\section{Advertising}

TMS technique is often used for product promotions.In recent years, TMS is gathering attention because it is possible to give strong impression to the consumers by conducting a product promotion along with a story by using multiple media platforms. The campaign,"\#Dropped” that Heineken led in 2013, is known as the successful case to use the TMS technique.For the campaign, TV CM, Website. SNSs, Web series (YouTube), Online game and ARG were used as media platforms. The videos of web series (YouTube) worked as the stem, and the stories and information related to the video were developed in the other media platforms (Fig.5). 

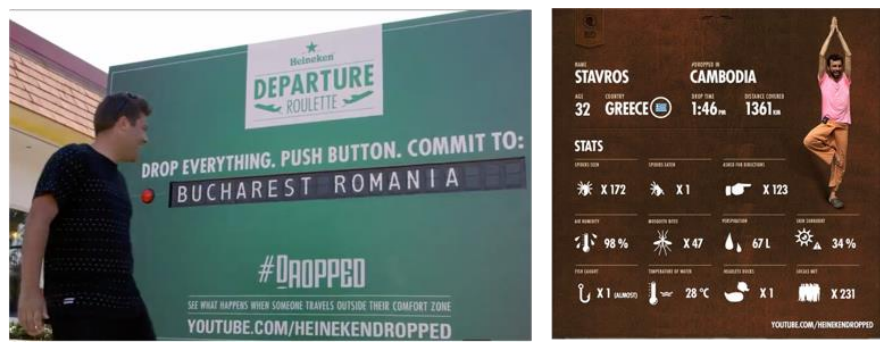

Fig.5.Heineken Campaign“\#Dropped” [7][8].

Each of media platforms had a specific role to get audience's attention.TV CM was used as an introductory measure to spread information, and SNSs were used to share chatting and information.In addition, Web series (You Tube) expanded the story and nurtured the audience's interests.Online game and ARG (including Real world) provided the audience with sense of immersion to the story world by delivering the story experience to them. "The best of video" summarized the best moments of the whole series.As the response to the Heineken campaign, the content of Departure Roulette (ARG) posted on Facebook in 2013, September $30^{\text {th }}$ got 49,471 'Like's, 9,310 comments, and the post was shared 4,677 times. This was the largest Social Networks effect of the campaign (Sánchez, 2014).Thus, regarding TMS project for the purpose of promotion, different media platforms have their own roles, and when all the functions work perfectly, the maximum number of views can be obtained.Information of how each media platform and story is connected and how they interact with each other can be visualized bya tree structure.In the way, the user can easily figure out the system of the project (Fig.6).

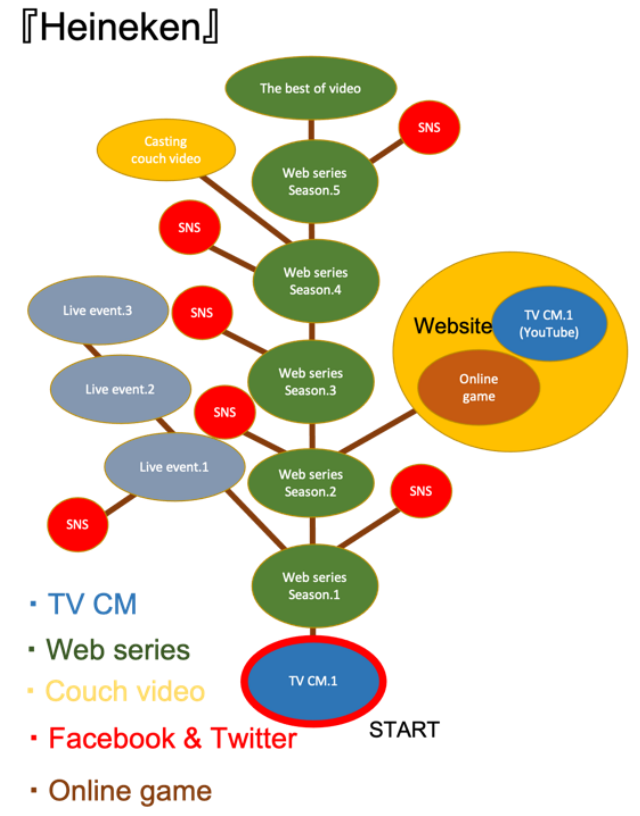

Fig.6.A tree structure of Heineken.

\section{SUMMARY AND FUTURE WORK}

TMS has a variety of information, which correlate each other complexly.By using multiple media platforms, one story is developed.The reason of complexity is the necessity of combining the story with the story experience and controlling the viewer's behavior to keep the story going as planned.In this study, weexamined what kind of effect can be obtained with the tree structure by applying the content elements ofsuccessful entertainment series, ARG, and promotion, for which TMS method is often used, to the tree structure. The TMS method is used in differentways in various fields. The projects in all disciplines have something in common such as developing a story using multiple media platforms, providing story experiences, and controlling the audiences' actions.By visualizing the positional relationship of content by using a tree structure and organizing information, users can efficiently work on the production and operation of those common parts.

As a future task, we will construct TMS-specific functions that accompany the tree structure. One example is the function that allows users to grasp the story.By tapping a content element in the tree structure, they can write the story of the content element.If we can implement the idea of such a function, the system will be able to further support creators working on TMS. 


\section{REFERENCES}

Robert Pratten 2015Getting Started with Transmedia Storytelling: A Practical Guide for Beginners, CreateSpace Independent Publishing Platform.

Henry Jenkins 2007 "Transmedia Storytelling 101" Henry JenkinsOfficial Blog, http://henryJenkins.org/blog/2007/03/transmedia_storytelling_101.html, 20,11,2020.

Yasushi Shinjo 2016 "Tree structure: Comprehensive subject 'Introduction to Information Technology that Supports the Network Society II"'Kikouzou: Sougoukamoku[Network shakai wo sasaerujouhougijutunyumon II](in Japanese),http://www.softlab.cs.tsukuba.ac.jp/ yas/gen/it-2016-11-21/, 28.12.2020.

Carmen Costa Sánchez, "Transmedia Storytelling, an ally of Corporate Communication: \#Dropped by Heineken case study", Communication \& Society / Comunicación y Sociedad, Vol. 27, n. 3, pp.127-150, 2014.

Star wars official website, https://www.starwars.com/, 3.12.2020.

Why so serious? Official website, "Investigative report - summer of 2008", https://www.whysoseriousredux.com/home.htm, 21.01.2021.

Heineken official Facebook account, https://www.facebook.com/heineken/videos/10152372085997355, 12.12.2020.

Heineken official Instagram account, https://www.instagram.com/p/bjBDKCwlMW/, 12.12.2020.

ARGinformation service, “Case study Film 'Dark Knight' ARG 'Why so serious?'”JireishoukaiEiga 'Dark knight' ARG 'Why so serious?’(in Japanese),https://arg.igda.jp/2012/07/argwhy-so-serious.html,22.12.2020.

Sean Guynes, Dan Hassler-Forest 2017 STAR WARS and the History of Transmedia Storytelling, Amsterdam University Press.

Christian Brieger, "Exploring New Communication Strategies for a Global Brand - Transmedia Storytelling and Gamification”, NHH Norwegian School of Economics, Norway, Mater thesis, 2013.

Steve Barrett 2008 “Aview from Steve Barrat: Happiness Factory offers a vision of media's future” Campaign,

https://www.campaignlive.co.uk/article/happiness-factory-offers-vision- medias- future/810206,22.10.2020.

Eric Spaulding, "Transmedia Storytelling: Principles, practices, and prototypes for designing narrative experiences with the audience", Carnegie Mellon University, U.S.A, Thesis, 2018.

Starlight Ruunnerentertaiment, Happiness "Open Happiness", https://starlightrunner.com/portfolio-items/coca-cola/, 03.11.2020.

Tosca Susan, Junichi Ishikawa, Akinori Nakamura, "A Tripartite Discussion: Transmedia Storytelling, Media-Mix, Cross-Media, and other Surrounding Terminology: Academic and Practice perspectives on their Usage in the West and The East", Journal of College of Image Arts and Sciences, Ritsumeikan University, Vol. 12, pp.45-57, 2019.

Henry Jenkins 2003 "Transmedia Storytelling: Moving characters from books to films to video games can make them stronger and more compelling." MIT Technology Review, https://www.technologyreview.com/s/401760/transmedia-storytelling/, 12.11.2020.

Junichi Ishikawa 2019, “Learning 'Tell in a game' technique from Transmedia storytelling 2019” Transmedia storytelling karamanabu 'Game demonogataru'shuhou 2019 (in Japanese)CEDEC, https://www.slideshare.net/elejun/2019-169957648, 12,10,2020. 